\title{
Preface
}

\section{The Complex World of Ventricular Preexcitation: Toward Precision Electrocardiology}

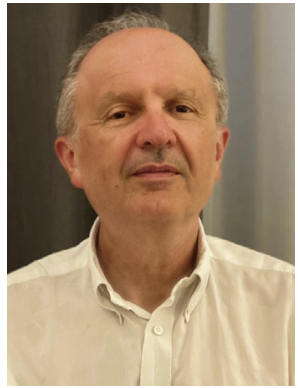

Giuseppe Bagliani, MD

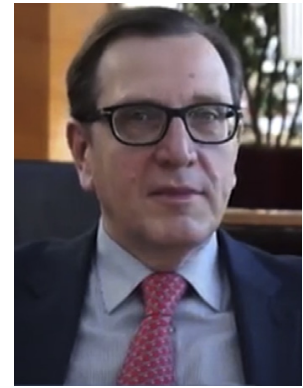

Roberto De Ponti, MD, FHRS Editors
This issue, the fourth consecutive publication edited by our group, follows "Reinterpreting the Basic ECG," "Clinical Aspects of Arrhythmias," and, last year, "The Rational Approach to Complex Arrhythmias."

This year the topic is ventricular preexcitation, which we have reviewed within the optic of "Precision Electrocardiology," the method of electrocardiogram (ECG) interpretation that has been the common thread for all the previous issues.

Ventricular preexcitation, with its multifaceted electrocardiographic expressions, is indeed a natural marvel potentially encompassing every electrophysiologic principle.

A normal conducting system in parallel with an accessory atrioventricular pathway both activated competing to activate the ventricles. From an unusual ECG in sinus rhythm, clearly demonstrating this competition, to a sudden covenant where the 2 conducting systems are joined by ventricles and atria in a macro-reentrant circuit. A sudden inversion of the direction of propagation can almost simultaneously but, at the same time,

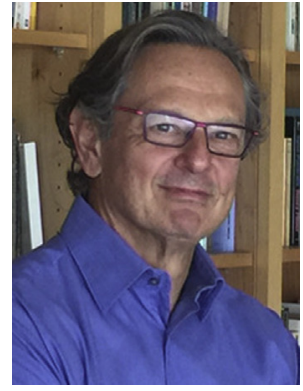

Fabio M. Leonelli, MD dramatically change the ECG appearance, requiring uncommon skills and knowledge for its immediate identification!

The macro-reentry can become even more complex depending on the properties of the AP, which can vary from pure muscle behavior to decremental conduction not dissimilar from the AVN.

And the same electrophysiologic pathway properties can change a common and relatively benign arrythmia, such as atrial fibrillation, into a killer by inducing ventricular fibrillation and sudden cardiac death.

Invasive EP study first and percutaneous catheter ablation have allowed us to understand the electrophysiology of AP and AP-mediated arrythmias and later effectively treat WPW patients.

In the last 20 years, catheter ablation has been shown to be a curative procedure for WPW patients; when performed by expert hands, this procedure can be considered safe and effective and is a first therapeutic choice in selected patients. 
Safe but not completely so; the question of risk stratification to select patients clearly benefiting from this procedure has not yet being completely resolved.

The structure of this issue is an attempt to review all these aspects of ventricular preexcitation and is divided into 3 different sections:

1. In the first part of the issue, we review in great detail the anatomic and electrophysiologic substrate generating the typical ECG pattern of preexcitation.

2. The second section focuses on the electrophysiologic mechanisms at the base of APmediated arrythmias. In these 2 sections, the ECG is the major point of reference.

3. Finally, the third section reviews the prognostic and therapeutic aspects of management of patients with clinical preexcitations.

This issue is meant for internists, clinical cardiologists, and electrophysiologists. Each article is complemented by a wide range of figures, including ECGs, endocardial tracings, and cartoons used as schematic explanations. This has been done in an attempt to clarify the entire range of AP's behaviors and to render the text useful for all the clinicians interested in ventricular preexcitation.

Our work has been at times difficult but very satisfactory; the ECG analysis we present, complemented by endocavitary recordings, has allowed us to appreciate again the usefulness of "Precision Electrocardiology" used as an interpretative key of complex electrophysiologic phenomena.

We would like to thank Dr Thakur and Dr Natale for their continue support of our endeavors and for allowing us to share our ideas with a wide audience.
This issue is dedicated to the memory of Professor Luigi Padeletti, friend, teacher, and mentor, who inspired and guided the writing of these issues.

Giuseppe Bagliani, MD Arrhythmology Unit

Cardiology Department

Foligno General Hospital

Via Massimo Arcamone

Foligno, Perugia 06034, Italy

Cardiovascular Disease Department University of Perugia Perugia, Italy

Roberto De Ponti, MD, FHRS

Department of Cardiology School of Medicine

University of Insubria

Viale Borri, 57, Varese Varese 21100, Italy

Fabio M. Leonelli, MD Cardiology Department James A. Haley Veterans' Hospital 13000 Bruce B Down Boulevard Tampa, FL 33612, USA

University of South Florida 4202 East Fowler Avenue Tampa, FL 33620, USA

E-mail addresses:

giuseppe.bagliani@tim.it (G. Bagliani) roberto.deponti@uninsubria.it (R. De Ponti) fabio.leonelli@va.gov (F.M. Leonelli) 